\title{
Correlation Between the Activities of Daily Living Assessment and Gross Motor Function Measures in Children with Spastic Cerebral Palsy
}

\author{
Hee-Joo You', Sun-Wook Park', Han-Suk Lee² \\ 'Department of Physical Therapy, Graduate School, Eulji University, Seongnam; ${ }^{2}$ Department of Physical Therapy, College of Health Science, Eulji \\ University, Seongnam, Korea
}

Purpose: This study was to identify the whether there is any correlation between Gross Motor Function Measures (GMFM) and Functional independence Measurement for children (WeeFIM) in order to identify a relevance of daily life motor with gross motor function of children with cerebral palsy.

Methods: Subjects were instructed to evaluated by an occupational therapist using WeeFIM (reliability 0.83-0.99) and by a physical therapist using GMFM (reliability 0.93). 10 male children and 13 female children among 23 children being diagnosed as cerebral palsy were targeted for this study. A correlation between GMFM and WeeFIM was analyzed with Kendall correlation.

Results: Participants showed significant correlation between GMFM and WeeFIM in all categories, except in the GMFM's walking. running.jumping category, standing category of GMFM and self care, sphincter control, communication category of WeeFIM. Lying-rolling and sitting and crawling kneeling area of GMFM showed a significant correlation with self care $(r=0.35,0.39,0.4)$, sphincter control $(r=0.45,0.43,0.61)$, mobility transfer $(r=0.44,0.36,0.64)$, locomotion $(r=0.41,0.35,0.74)$, communication $(r=0.4,0.44,0.51)$, and social cognition $(r=0.43,0.51,0.64)$ area of WeeFIM $(p<0.05)$. Standing of GMFM and mobility transfer $(r=0.74)$, locomotion $(r=0.47)$ and social cognition $(r=0.4)$ area of WeeFIM showed a significant correlation $(p<0.05)$.

Conclusion: These findings indicated significant correlation between activity of daily living (ADL) and gross motor function of children with cerebral palsy. However, because there are a few results that are not significant, both assessment tools should be used for evaluations so that treatment can be achieved with an accurate assessment and establish a therapeutic plan.

Keywords: Cerebral palsy, WeeFIM, GMFM, Activities of Daily Living

\section{INTRODUCTION}

The incidence rate of cerebral palsy among children in South Korea is 3.2 per 1,000 children, and has been increasing in recent years. ${ }^{1}$ Despite advancements in modern medical science and the health management industry, rates of cerebral palsy (CP) continue to rise. ${ }^{2}$ These children with cerebral palsy experience various difficulties, including dystonia, abnormal posture, and involuntary movements. ${ }^{3}$ Spastic quadriplegia, in particular, brings about multiple disabilities involving physical, cognitive, perceptive, and learning impairments, and even when treatments are given, patients require

Received Nov 25, 2015 Revised Dec 22, 2015

Accepted Dec 24, 2015

Corresponding author Han-Suk Lee

E-mail leehansuk21@naver.com long treatment durations. Therefore, studies on children with spastic quadriplegia serve a very significant purpose. ${ }^{4}$ Among the activities of daily living (ADL) in children with cerebral palsy, most patients commonly require assistance with dressing, washing, and eating. ${ }^{5}$ if children with cerebral palsy are unable to perform these basic activities due to physical challenges, then it becomes difficult for them to receive positive feedback. These can lead to psychological along with physical problems, as well as isolation from the rest of society.

Therefore, it is necessary for physical therapists to focus on the improving the performance of the ADL among children with cerebral

Copylight (C)2015 The Korea Society of Physical Therapy

This is an Open Access article distribute under the terms of the Creative Commons Attribution Non-commercial License (Http:// creativecommons.org/license/by-nc/3.o.) which permits unrestricted non-commercial use, distribution, and reproduction in any medium, provided the original work is properly cited. 
palsy. However, most therapists focus on treating gross motor function measure (GMFM) and do not consider the importance of improving the ADL performance. If the training programs that are implemented after GMFM include the assessment of the ADL and analysis of their correlation in a sufficient manner, then independent life and acclimation into society will be easier for children with cerebral palsy. The GMFM is a highly valid that examines dysfunction in children with cerebral palsy and is widely used in Korea. ${ }^{6}$ Tools for assessing ADL also include the Wee Functional Independence Measure (WeeFIM) and Modified Barthel Index (MBI). The WeeFIM, in particular, is an ADL assessment tool that emphasizes the cognitive aspects. Recent attempts to analyze the associations among these assessment tools still need further evaluation. The $\mathrm{Um}^{7}$ and $\mathrm{Kim}^{8}$ studied the correlations between the quality of the upper extremity function and GMFM according to area and severity of disability in children with spastic cerebral palsy. However, ADL, which is a practical issue for children with cerebral palsy, was not included in the analysis. Moreover, in the study by Go, ${ }^{9}$ which examined the correlations of ADL performance with manual ability and GMFM in children with cerebral palsy, it was reported that GMFM, upper extremity function assessment, and ADL performance assessment were significantly correlated with each other. Therefore, it is necessary to identify the correlation of ability of ADL and GMFM. Most of physical therapist has used the MBI to evaluate the ability of ADL in the clinic. However, MBI did not consider the cognitive aspect. Therefore, ADL assessments that take into account the cognitive aspects, such as using the WeeFIM, are still needed. Accordingly, this used the WeeFIM assessment tool to examine the correlation between ADL and GMFM in children with spastic cerebral palsy. Our aim was to identify the correlation of ability of ADL and GMFM and suggest the guideline to establish the clinical treatment plans.

\section{METHODS}

\section{Subjects}

Twenty-three children with spasticity quadriplegia cerebral palsy were recruited in this study. The subjects did not medical intervention like surgery during the evaluation. General characteristics of the subjects were shown in Table 1.
Table 1. General Characteristics of the subjects

\begin{tabular}{llcc}
\hline Classification & & Number & $\%$ \\
\hline Gender & Male & 11 & 47.8 \\
\multirow{4}{*}{ Age } & Female & 12 & 52.2 \\
& $1-2$ & 1 & 4.3 \\
& $3-4$ & 11 & 47.8 \\
& $5-6$ & 10 & 43.4 \\
GMFM Level & 7-8 & 1 & 4.3 \\
& 1 Level & 1 & 4.3 \\
& 2 Level & 3 & 13 \\
& 3 Level & 2 & 8.6 \\
& 4 Level & 0 & 0 \\
& 5 Level & 17 & 73.9 \\
\hline
\end{tabular}

\section{Measurement}

For the assessment, measurements were taken from the same location by two physical therapists that was in their above 3 years of training with previous GMFM assessment experience. All items were measured in person by the same physical therapist, and for better understanding of the items being tested, demonstrations were given and toys were used in order to perform the activities that corresponded to the test item. Approximately 1 hour was required for the testing. The WeeFIM assessment was performed by two occupational therapists who were in their 2 nd or higher year of training. Assessments of the performance of ADL by the children and each of the categories presented were conducted. We used the toy to induce an interest or motivation to evaluate each region of assessment. Assessment of WeeFIM takes about $30 \mathrm{~min}$.

\section{1) Wee functional independence measure (WeeFIM)}

WeeFIM was developed for the functional assessment of children aged 6 months to 7 years ${ }^{10}$ and is composed of 2 domains including motor domain and cognitive domain. It was divided into 6 categories including self care, sphincter control, mobility, locomotion, communication, and social cognition. It measures 18 items in 6 categories on a 7-point scale, with a minimum score of 18 points indicating the inability to perform any ADL, and a maximum score of 126 points indicating normal ADL performance.

\section{2) Gross Motor Function Measurement 66 (GMFM66)}

The GMFM 66 is a condensed version of the GMFM 88, which was developed as a more efficient assessment, by taking the 88 items from the GMFM 88 and revising those to 66 items according to the 
degree of difficulty, and through the Rash model. ${ }^{11}$

The GMFM 66 is composed of 66 items in 5 categories that consist of activities involving lying and rolling, sitting, crawling and kneeling, standing and walking, running, and jumping. Since each item uses a 4-point scale, it is possible to arrive at a relatively accurate score even if all categories are not considered.

\section{Statistical analysis}

Data obtained in the present study were analyzed using the statistical program, SPSS 21.0, and the general characteristics were analyzed by descriptive statistics, while the correlation between GMFM and WeeFIM was analyzed using Kendall's correlation. The level of statistical significance was set to 0.05 .

\section{RESULTS}

In looking at the correlations between GMFM and WeeFIM, the GMFM's lying and rolling category showed significantly positive correlations with WeeFIM's self care $(\mathrm{r}=0.35, \mathrm{p}<0.05)$, sphincter control $(\mathrm{r}=0.45, \mathrm{p}<0.01)$, mobility $(\mathrm{r}=0.44, \mathrm{p}<0.05)$, locomotion $(\mathrm{r}=0.41, \mathrm{p}<0.05)$, communication $(\mathrm{r}=0.4, \mathrm{p}<0.05)$, and social cognition $(r=0.43, p<0.05)$ categories. Moreover, GMFM's sitting category showed significantly positive correlations with WeeFIM's self care $(\mathrm{r}=0.39, \mathrm{p}<0.05)$, sphincter control $(\mathrm{r}=0.43, \mathrm{p}<0.05)$, mobility $(\mathrm{r}=0.36, \mathrm{p}<0.05)$, locomotion $(\mathrm{r}=0.35, \mathrm{p}<0.05)$, communication $(\mathrm{r}=0.44, \mathrm{p}<0.05)$, and social cognition $(\mathrm{r}=0.51, \mathrm{p}<0.01)$ categories. Furthermore, GMFM's crawling and kneeling category also showed significantly positive correlations with WeeFIM's self care $(r=0.4$, $\mathrm{p}<0.05)$, sphincter control $(\mathrm{r}=0.61, \mathrm{p}<0.01)$, mobility $(\mathrm{r}=0.64$, $\mathrm{p}<0.01)$, locomotion $(\mathrm{r}=0.74, \mathrm{p}<0.01)$, communication $(\mathrm{r}=0.51$, $\mathrm{p}<0.01)$ and social cognition $(\mathrm{r}=0.64, \mathrm{p}<0.01)$ categories. Meanwhile, GMFM's standing category showed significantly positive correlations with WeeFIM's mobility $(\mathrm{r}=0.74, \mathrm{p}<0.01)$, locomotion $(\mathrm{r}=0.47, \mathrm{p}<0.05)$, and social cognition $(\mathrm{r}=0.4, \mathrm{p}<0.05)$ categories. However, significant correlations were not seen between GMFM's standing category and WeeFIM's self care $(r=0.96)$, sphincter control $(r=0.32)$, and communication $(r=0.3)$, as well as between GMFM's walking, running, and jumping category and WeeFIM's self care $(r=-341)$, sphincter control $(r=0.32)$, mobility $(r=-0.08)$, communication $(r=0.21)$, and social cognition $(r=0.21)$ categories (Table 2). There were significant correlations in all areas, except GMFM's standing category and WeeFIM's self care, sphincter control, and communication, as well as between GMFM's walking, running, and jumping category and WeeFIM's self care, sphincter control, mobility, communication, and social cognition, categories.

\section{DISCUSSION}

Cerebral Palsy (CP) is a syndrome that affects movements and postural development due to non-progressive damage occurring in the brain of a growing fetus or infant that restricts movements and functional activities. ${ }^{12}$ In recent years, advancements in the medical care of premature and very low birth weight infants have increased the survival rates of preterm infants. Consequently, this has also increased the possibility of these infants developing cerebral palsy. ${ }^{13}$ The biggest challenge that these children with cerebral palsy encounter is their inability to independently perform ADL. To enhance the performance of these ADL, it would be necessary to examine the relationship between ADL and GMFM for potential use in subsequent treatments. Accordingly, the present study examined the correlation between ADL and GMFM and aimed to provide the foundation for a clinical treatment plan.

$\mathrm{Go}^{9}$ examined the correlations between the performance of $\mathrm{ADL}$ with manual ability and GMFM in children with cerebral palsy and

Table 2. Correlation between WEEFIM and GMFM of children with cerebral palsy

\begin{tabular}{|c|c|c|c|c|c|c|c|}
\hline & & \multicolumn{6}{|c|}{ WEEFIM } \\
\hline & & Self care & Sphincter control & Mobility & Locomotion & communication & Social cognition \\
\hline G & Lying $\cdot$ Rolling & $0.35^{*}$ & $0.45^{* *}$ & $0.44^{*}$ & $0.41^{*}$ & $0.4^{*}$ & $0.43^{*}$ \\
\hline M & Sitting & $0.39^{*}$ & $0.43^{*}$ & $0.36^{*}$ & $0.35^{*}$ & $0.44^{*}$ & $0.5^{* *}$ \\
\hline $\mathrm{F}$ & Crawling $\cdot$ Kneeling & $0.4^{*}$ & $0.61^{* *}$ & $0.64^{* *}$ & $0.74^{* *}$ & $0.51^{* *}$ & $0.64^{* *}$ \\
\hline \multirow[t]{2}{*}{ M } & Standing & 0.96 & 0.32 & $0.74^{* *}$ & $0.47^{\star}$ & 0.3 & $0.4^{*}$ \\
\hline & Gait· Running · Jumping & -0.34 & 0.32 & -0.08 & -0.08 & 0.21 & 0.21 \\
\hline
\end{tabular}

${ }^{*} p<0.05,{ }^{* *} p<0.01$ 
demonstrated that ADL (MBI) was highly correlated with GMFM. However, GMFM has a greater degree of significantly positive correlation with manual ability than with ADL. In the present study, the GMFM and ADL (WeeFIM) were highly correlated to each other. It is believed that these results were due to the development of GMFM leading to an increase in the motivation for performing ADL and enhancement of fine motor functions. According to this study, there were correlations in most areas; however there were especially high correlations in areas of crawling-kneeling of GMFM and WeeFIM.

In the present study, GMFM activities, such as crawling, were correlated with self care, sphincter control, and communication. Crawling can be performed better with reduction of the grasping reflex and improvement in the fine motor function of the hands. Such improvement in fine motor function of the hands can also enhance ADL such as self care. Moreover, the ability to perform GMFM activities such as crawling improves the child's confidence level and increases the motivation to accomplish something. This subsequently leads to the desire for more active communications and results in improved communication. Lee ${ }^{14}$ reported that the GMFM in children with spastic diplegia was highly correlated with social maturity. The present study also showed a high correlation between cognitive development and GMFM. It is believed that as GMFM developed, there were more opportunities for movement, which led to more opportunities for social exploration. As a result, an increase in the range and amount of motor learning by the child was observed which led to improved cognitive development.

However, correlations that were not significant were seen between GMFM's standing category and WeeFIM's self care, sphincter control, and communication categories, as well as between GMFM's walking, running, and jumping category and WeeFIM's self care, sphincter control, mobility, communication, and social cognition categories. These results are believed to be due to the specific characteristics of the subjects' disability type. $\mathrm{Um}^{7}$ compared the correlations between GMFM and quality of upper extremity function assessment according to the area and severity of disability in children with spastic cerebral palsy, and reported that correlations were seen according to the area of paralysis and severity of disability. However, as the disability became more severe, correlations became weaker. Moreover, in his research it was stated that there were limitations in comparisons of each subcategories. This was because, due to reduction of functionality which is characteristic of quadriplegia, the gross and fine motor skill levels were already very low making it impossible to perform subcategories for each function. Also it was stated that for all paralyzed areas of test subjects, all 10 children with spastic quadriplegia were at disability levels of 4 or 5 , and were unable to perform most upper extremity function and gross motor functions; therefore it was difficult to find statistical significance. Comparing this to our research, it seems that there is some relevance because there seems to be a correlation between upper extremity functions and every day activities. In the present study, children with spastic quadriplegia who were selected as subjects of the study had a greatly diminished motor status, in comparison to children with other types of cerebral palsy, and were thus were limited in the sub-items that they can actually perform. In other words, it is believed that because categories. According to Oh's ${ }^{15}$ study, spastic cerebral palsy includes increased factors of problem in standing and walking. The limitations of the present study included the fact that due to the small sample size, the results cannot be generalized. Since only children with spastic quadriplegia were included in the study, the comparison of the high-level categories, such as GMFM's standing and walking, running, and jumping, was inappropriate. Therefore, it is necessary for future studies to subdivide the patients according to the types of cerebral palsy and severity of disability, when examining the correlations between ADL and GMFM.

During the examination of the correlations between ADL and GMFM in children with spastic quadriplegic cerebral palsy, there were significant correlations between GMFM and WeeFIM in all categories, except in the GMFM's walking, running, jumping category, standing category of GMFM and self care, sphincter control, communication category of WeeFIM. This research shows that, looking at the significant correlation between ADL and gross motor functions, there seems to be a correlation between assessment tools. However, because there are a few results that are not significant, both assessment tools should be used for evaluations so that treatment can be achieved with an accurate assessment. Since the correlations were analyzed in a sufficient manner, then independent living and acclimation into society will be easier for children with cerebral palsy. GMFM is a highly valid method for examining dysfunction in children with cerebral palsy and is widely used in Korea. Tools for assessing ADL also include the Wee Functional Independence Measure. 


\section{REFERENCES}

1. Park EY. Verification of validity on the manual ability classification system in children with spastic cerebral palsy. International JOURNAL OF CONTENTS. 2010;10(1):317-32.

2. Yun MJ, Lee WH, Lee JS et al. The relationship between degree of stress and quality of life in mothers of children with cerebral palsy. J Kor Soc Phys Ther. 2012;24(3):191-7.

3. Hagberg B, Hagberg G, Olow I. The changing panorama of cerebral palsy in sweden 1954-70. I. Analysisof the general changes. Acta Paediatr Scand. 1975;64:187-92.

4. Kim GG. Case-control study of risk factors affecting cerebral palsy with spastic quadriplegia. Journal of Korean Society of Occupational Therapy. 2000;8(1):77-924.

5. Yoon YG. The effects of the ADL tranining on the self-car skills for the children with cerebral palsy. Dissertation of Master's Degree. Daegu University. 2001.

6. Lee GO. Lifelong learning outcomes determinants factors of disadvantaged groups. Graduate School Chonnam National University. Dissertation of Doctorate Degree. 2014.

7. Um JS. The correlation between gross motor function measure and quality of upper extremity skills test according to the distribution and severity of disability in children with spastic cerebral palsy. Dissertation of Master's Degree. Hanyang University. 2013.
8. Kim SW. Correlation of quality of upper extremity skills test and gross motor function measure related age in the children with spastic diplegia. Yongin University. . Dissertation of Master's Degree. 2008.

9. Go JY. A study on functional and quality of life of preschoolers with cerebral palsy. Sahmyook University. Dissertation of Master's Degree. 2008.

10. Msall ME, DiGaudio KM, Duffy LC. "Use of functional assessment in children with developmental disabilities," Phys Med Rehabil Clin N Am. 1993;4:517-27.

11. Alotaibi M. The use of constant time delay in the acquisition of incidental learning when teaching functional sight word/phrases recognition to students with moderate and severe disabilities. Dissertation of Doctorate Degree. 2014

12. Choi HJ, Kim KJ, Nam KJ. The effects of a horseback riding simulation exercise on the spinal alignment of children with cerebral palsy. J Kor Soc Phys Ther. 2014;26(3):209-15.

13. Vincer MJ, Allen AC, Joseph K et al. Increasing prevalence of cerebral palsy among very preterm infants: a population-based study. Pediatrics. 2006;118(6):1621-6.

14. Lee JG. The relevance between gross motor function measurement (GMFM) and the spatiotemporal parameters of gait in children with cerebral palsy. yonsei University. Dissertation of Master's Degree. 2002.

15. Oh TY. The Comparison of different of normal development walking and walking characteristics of children with spastic cerebral palsy. J Kor Soc Phys Ther. 1997;9(1):195-204. 